\title{
Unlocking the Nature of the Phonological-Deep Dyslexia Continuum: The Keys to Reading Aloud Are in Phonology and Semantics
}

\author{
Jenni Crisp ${ }^{1,2}$ and Matthew A. Lambon Ralph ${ }^{3}$
}

\begin{abstract}
It has been argued that normal reading and acquired dyslexias reflect the role of three underlying primary systems (phonology, semantics, and vision) rather than neural mechanisms dedicated to reading. This proposal is potentially consistent with the suggestion that phonological and deep dyslexia represent variants of a single reading disorder rather than two separate entities. The current study explored this possibility, the nature of any continuum between the disorders, and the possible underlying bases of it. A case series of patients were given an assessment battery to test for the characteristics of phonological and deep dyslexia. The status of their underlying phonological and semantic systems was also investigated. The majority of participants exhibited many of the symptoms
\end{abstract}

\section{INTRODUCTION}

The "triangle" connectionist model of reading (Plaut, McClelland, Seidenberg, \& Patterson, 1996) is based on the notion that reading, like a range of other language tasks (e.g., Joanisse \& Seidenberg, 1999), can best be understood by modeling the individual competence of and degree of interaction between primary neural systems such as semantics, phonology, and vision (Patterson \& Lambon Ralph, 1999). There are two key sequelae to this approach: First, disorders of reading can be understood in terms of the disruption to one or more primary systems, thus obviating any need to postulate the existence of, and damage to, procedures specific to reading. Second, where such disruption to primary systems occurs, performance will be impaired across a range of both reading and nonreading tasks because all these language activities are underpinned by the same systems.

To date, much of the work adopting this approach has focused on surface dyslexia. This is an acquired reading disorder characterized by more accurate reading of words with predictable than exception/inconsistent spelling-to-sound correspondences. In addition, the pa-

\footnotetext{
${ }^{1}$ North Tyneside Primary Care Trust, ${ }^{2}$ University of Newcastle, ${ }^{3}$ University of Manchester
}

associated with deep dyslexia whether or not they made semantic errors. Despite wide variation in word and nonword reading accuracy, there was considerable symptom overlap across the cohort and, thus, no sensible dividing line to separate the participants into distinct groups. The patient data indicated that the deep-phonological continuum might best be characterized according to the severity of the individual's reading impairment rather than in terms of a strict symptom succession. Assessments of phonological and semantic impairments suggested that the integrity of these primary systems underpinned the patients' reading performance. This proposal was supported by eliciting the symptoms of deep-phonological dyslexia in nonreading tasks.

tients' errors reflect the typical pronunciation of the orthographic elements (e.g., PINT is read as though it rhymed with "mint": Jefferies, Lambon Ralph, Jones, Bateman, \& Patterson, 2004; Patterson \& Behrmann, 1997; Patterson \& Hodges, 1992). Using the triangle model of reading, Plaut et al. (1996) were able to demonstrate that damage to semantic representations, or the reduction in their influence on phonological activation, leads to surface dyslexia. This pattern mirrors that found in patients with semantic dementia, a neurodegenerative disorder leading to a progressive yet selective deterioration in conceptual knowledge (Hodges, Patterson, Oxbury, \& Funnell, 1992; Patterson \& Hodges, 1992). The association between semantic impairment and surface dyslexia across patients is very strong with only one exception reported in the literature (Cipolotti \& Warrington, 1995). Using a case-series design, a number of studies have demonstrated that exception word reading accuracy is correlated with the degree of semantic impairment (Jefferies et al., 2004; Patterson \& Hodges, 1992). This relationship has also been demonstrated on a by-items basis such that reading accuracy for individual words can be predicted by the status of the patients' remaining conceptual knowledge for those items (Graham, Hodges, \& Patterson, 1994).

As noted above, the primary systems hypothesis (Patterson \& Lambon Ralph, 1999) predicts that semantic 
impairment should also produce impairments in a predictable range of language and nonlanguage activities (Patterson et al., 2006). This includes semantic impairment leading to profound anomia (Lambon Ralph, McClelland, Patterson, Galton, \& Hodges, 2001), poor object and lexical decision (Rogers, Lambon Ralph, Hodges, \& Patterson, 2004), surface dysgraphia in addition to surface dyslexia (Graham, Patterson, \& Hodges, 2000), and more errors on irregular than regular verbs when patients are required to produce or recognize the correct past tense in a sentence-completion task (Patterson, Lambon Ralph, Hodges, \& McClelland, 2001). In all these different domains, the level of impairment has been found to be related to the degree of semantic deterioration (Patterson et al., 2006).

Patterson and Lambon Ralph (1999) also explored the possibility that other acquired dyslexias-pure alexia/letter-by-letter reading, phonological and deep dyslexia-might similarly be understood in terms of disruption to one or more of the other primary neural systems. As for semantic impairment, not only should each type of primary system damage lead to a type of acquired dyslexia but this same damage should also give rise to principled, coherent patterns of impairment across a range of both reading and nonreading activities. The current study explores Patterson and Lambon Ralph's hypothesis in relation to phonological and deep dyslexia.

The literature on phonological and deep dyslexia is dominated by single case studies (for reviews, see Lambon Ralph \& Graham, 2000; Berndt, Haendiges, Mitchum, \& Wayland, 1996). The cardinal diagnostic feature of deep dyslexia is the production of semantic paralexias (e.g., sergeant is read as "soldier"). This is accompanied by a number of other co-occurring symptoms, including (1) visual (e.g., badge $\rightarrow$ "bandage") and derivational errors (e.g., edition $\rightarrow$ "editor"); (2) abolished or very poor reading of nonwords (novel, pronounceable letter strings such as dake, gat, etc.); (3) an effect of the "part of speech" on reading accuracy-patients find nouns easier to read than adjectives, which in turn are easier than verbs, and function words are particularly difficult; and (4) an effect of imageability on reading accuracy-patients read concrete words (such as winter, stream, kitchen, etc.) more successfully than abstract words (e.g., reality, motivation, etiquette, etc.) The central feature of phonological dyslexia (Beauvois \& Derouesne, 1979) is a lexicality effect such that reading is more accurate for words than nonwords. This typically reflects a relative impairment rather than a complete abolition of nonword reading, whereas performance on words can be close to ceiling in some cases (Glosser \& Friedman, 1990). Reading errors include phonologically related responses and lexicalization errors (e.g, gat $\rightarrow$ "gate"). At least some phonological dyslexics demonstrate effects of imageability and part-of-speech effect, and sometimes make derivational and visual errors. Semantic paralexias, by definition, are not produced.
Although deep and phonological dyslexia have traditionally been viewed as distinct disorders, it may be that they simply reflect different degrees of impairment. Indeed, it is evident even in early case reports that questions were being raised over how distinct the two disorders actually were. When Beauvois and Derouesne (1979) published the first reported case of phonological dyslexia, they described three distinct features in the patient's $(R G)$ reading profile. First, they found that $R G$ presented with a classical dissociation between normal word reading yet impaired nonword performance. Second, they argued that he had a phonological-level problem in the absence of visual processing difficulties or other output difficulties. Finally, they noted that RG's reading problems occurred in the context of apparently normal oral expression, comprehension, writing, and spelling aloud. They suggested, therefore, that RG was significantly different from other reported cases (notably the deep or "phonemic" dyslexics of Shallice \& Warrington, 1975; Marshall \& Newcombe, 1973) in that he presented with a reading difficulty without a concomitant aphasia and, in particular, he made no semantic reading errors. Beauvois and Derouesne thus proposed the new term, "phonological dyslexia," to describe RG. Even in this first report, however, these researchers added the proviso that RG may have been a "spectacularly" pure case and suggested that phonological dyslexia more usually co-occurs with other disturbances that give rise to deep dyslexia. In the second case report of phonological dyslexia, Patterson (1982, p. 84) explicitly highlighted the overlap between patient AM's presentation and the clinical picture of deep dyslexia: "when I first saw AM ... I had never heard of phonological dyslexia and I judged him to be a very mild deep dyslexic."

More recently, the work of Friedman and her colleagues has focused on the two disorders and their possible overlap. Glosser and Friedman (1990) introduced the notion of a continuum in which deep dyslexia is the endpoint. Friedman (1996) presented two lines of evidence in favor of this continuum hypothesis. First, she reviewed five patients whose acquired reading disorders had evolved from deep to phonological dyslexia during the course of recovery. In each of these cases, semantic paralexias were the first symptom to change (a partial or total reduction). Resolution of other symptoms then followed to varying extents. Nonword reading was always the last symptom to change and there were no reports in which a complete recovery occurred. This longitudinal pattern of recovery clearly suggests that the two disorders are not independent. The second line of evidence was based on a severity-linked succession of symptoms. Friedman reviewed 11 published case reports of phonological/deep dyslexia and noted a consistent progression of symptoms. Friedman placed considerable emphasis on the order in which reading symptoms emerged and suggested that the continuum 
hypothesis was supported by this predictable succession of symptoms (see Table 1).

In Table 1 , the columns denote the symptoms of deep dyslexia that enter from the left-hand side as severity increases and exit from the right-hand side as recovery occurs. The first line represents a person with "classic" deep dyslexia, whereas the last of these represents someone with very mild phonological dyslexia. Friedman argued that this symptom succession was relatively fixed. There were, for example, no patients in the data she reviewed with symptom combinations such as those in the two bottom rows in Table 1 (e.g., an effect of imageability without a part-of-speech effect). Likewise, Friedman suggested that if a patient makes semantic paralexias then all four other symptoms should be present and that imageability effects are always the penultimate symptom.

What are the bases of the continuum between phonological and deep dyslexia? In a way that is consistent with the later primary systems hypothesis (Patterson \& Lambon Ralph, 1999), Glosser and Friedman (1990, p. 353) argued that the "apparent clinical continuity between the two reading disorders suggests the two forms of alexia may be due to disruption in common neurolinguistic systems." Although one might expect the notion of a severity-based continuum to imply variation along a single underlying dimension, Friedman (1996) went on to suggest that the continuum could be understood in terms of two coexisting impairments. First, she argued that there is a disruption of the orthographic-phonological connections. This accounts for the primary symptom of phonological dyslexia, the nonword reading difficulty-because nonwords can only be read by this nonsemantic pathway. Second, Friedman suggested that a semantic impairment accounts for the remaining dyslexia symptoms: the occurrence of derivational errors and a part-of-speech effect reflecting inher- ently poor support from the semantic representations of functors and bound morphemes, and other dyslexia symptoms (imageability effect, semantic paralexias), likewise occurring when the semantic system is impaired or stressed.

Whereas Friedman placed emphasis on a combination of impaired orthography $\rightarrow$ phonology and semantic deficit to explain the symptoms of phonological-deep dyslexia, from the perspective of phonological dyslexia other authors have argued that the reading impairment can be explained in terms of a single, more general (nonreading) phonological deficit (Patterson, Susuki, \& Wydell, 1996; Patterson \& Marcel, 1992). From this perspective, phonological representations are conceived as being weak or underactivated, and lexicality effects arise because (a) the phonological system has never experienced nonwords and (b) unlike words, nonwords cannot benefit from additional semantic support. It is possible that this premise could be extended to explain the other dyslexic symptoms. For example, if word reading benefits from semantic representations, then the effectiveness of this phonological-semantic interaction will be graded by intrinsic factors such as imageability. The natural sequitur of this hypothesis is that a generalized phonological deficit is inevitably manifested beyond poor nonword reading and indeed beyond the reading process itself. In a special issue of the journal Cognitive Neuropsychology (Coltheart, 1996), all 17 cases of phonological dyslexia performed outside the normal range on nonreading phonological tasks. Furthermore, Patterson and Marcel (1992) demonstrated that the cardinal feature of phonological dyslexia-a lexicality effect-can be observed in a range of nonreading tasks. Other studies have shown that even semantic errors can be elicited from deep dyslexics in nonreading tasks when greater demands are placed on their language system (e.g., by requiring multiword or

Table 1. Friedman's Severity-based Symptom Succession

\begin{tabular}{|c|c|c|c|c|c|c|}
\hline Patient & $\begin{array}{l}\text { Poor Nonword } \\
\text { Reading }\end{array}$ & Visual Errors & $\begin{array}{l}\text { Noun > } \\
\text { Functor }\end{array}$ & $\begin{array}{l}\text { Noun }> \\
\text { Verb }\end{array}$ & $\begin{array}{c}\text { Concrete }> \\
\text { Abstract }\end{array}$ & $\begin{array}{c}\text { Semantic } \\
\text { Errors }\end{array}$ \\
\hline \multicolumn{7}{|l|}{ Expected Pattern } \\
\hline \multirow[t]{5}{*}{ Severe } & $\checkmark$ & $\checkmark$ & $\checkmark$ & $\checkmark$ & $\checkmark$ & $\checkmark$ \\
\hline & $\checkmark$ & $\checkmark$ & $\checkmark$ & $\checkmark$ & $\checkmark$ & $x$ \\
\hline & $\checkmark$ & $\checkmark$ & $\checkmark$ & $\checkmark$ & $x$ & $x$ \\
\hline & $\checkmark$ & $\checkmark$ & $\checkmark$ & $x$ & $x$ & $x$ \\
\hline & $\checkmark$ & $\checkmark$ & $x$ & $x$ & $x$ & $x$ \\
\hline Mild & $\checkmark$ & $x$ & $x$ & $x$ & $x$ & $x$ \\
\hline \multirow[t]{2}{*}{ Unexpected Pattern } & $\checkmark$ & $\checkmark$ & $x$ & $x$ & $\checkmark$ & $x$ \\
\hline & $\checkmark$ & $\checkmark$ & $x$ & $\checkmark$ & $x$ & $x$ \\
\hline
\end{tabular}


delayed repetition: Allport, personal communication; Beland \& Mimouni, 2001).

The ability to assess the notion of a phonologicaldeep dyslexia continuum and the possible roles of phonological and semantic representations in explaining these acquired dyslexia is severely limited by the fact that the current literature is dominated by singlecase studies (with the exception of Berndt et al., 1996; Friedman, 1996). In contrast, the role of semantic impairment in explaining surface dyslexia plus other language and nonlanguage tasks has been confirmed by adopting a case-series methodology. While preserving the detail of single-case investigations, this approach allows direct comparisons between patients and thus the exploration of both qualitative and quantitative differences across the series of patients (Lambon Ralph, 2004; Lambon Ralph, Moriarty, \& Sage, 2002; Lambon Ralph et al., 2001). The current study used a case series of phonological-deep dyslexics (the largest to date) in order to address four research questions: (1) How strong is the link or overlap between phonological and deep dyslexia? (2) Is phonological-deep dyslexia accompanied by phonological/semantic deficits? (3) Can we, for the first time, demonstrate that the degree of these underlying primary system impairments predicts the patients' dyslexia symptoms? (4) Can the symptoms associated with their reading deficits be elicited in nonreading tasks as the primary systems hypothesis would predict?

\section{RESULTS}

\section{Assessing the Phonological-Deep Dyslexia Continuum}

The participants were tested using a battery designed to assess for a range of dyslexia symptoms including poor nonword reading, the production of paralexic errors (semantic, derivational, visual/phonological), an imageability effect, a length effect, a part-of-speech effect, and a consistency/regularity effect. Tests selected to quantify (nonreading) phonological and semantic impairments were also used. The results of the reading assessments from the 12 participants are shown in Table 2 and the rate of different types of reading error are summarized in Table 3. The participants are ordered from left to right by increasing word reading accuracy in all the tables and figures.

Consistent with the inclusion criteria, all participants performed outside the normal range on nonword reading. This was accompanied by a considerable range of word reading skills; some participants performed within the normal range on some word reading tests (e.g., DB, $\mathrm{TH})$, although none were completely unimpaired. The performance of others (e.g., LR, MM, RJ) was at or close to floor. All participants made at least some derivational errors (e.g., clouds $\rightarrow$ "cloudy") and some visual errors (e.g., winter $\rightarrow$ "window," value $\rightarrow$ "valley"). No significant part-of-speech effects were found using the Psycholinguistic Assessments of Language Processing in Aphasia (PALPA) grammatical class assessment (Test 32: Kay, Lesser, \& Coltheart, 1992). Given that this may have resulted from a lack of power in this test, we conducted a retrospective analysis including all words read aloud by the participants. This highlighted a significant effect of grammatical class in 6 of 12 participants. All but one of the participants (DB) exhibited an imageability effect in word reading. In summary (see Table 4) most, if not all, of the symptoms traditionally associated with deep dyslexia were present in all participants, although only LR, MM, RJ, and NS produced a significant proportion of semantic paralexias. It is clear from Table 4 that there is considerable overlap of dyslexia symptoms across the case series. This finding provides strong support for the contention that phonological and deep dyslexia are intimately related and are not two separate, independent disorders. Indeed, there is no obvious dividing line between the two disorders. There was limited support for Friedman's succession of symptoms (those that fit the pattern and those that do not are denoted by the footnote symbols a and $b$, respectively). On the positive side, semantic errors were associated with the poorest readers (the rate of semantic errors is correlated with word $[r=-.82, p=.001]$ and nonword $[r=.-67$, $p=.002]$ reading accuracy). Half of the participants, however, do not fit the predicted pattern in other regards. In particular, we found that all participants (bar one) demonstrated an effect of imageability on reading accuracy - a characteristic that is predicted to be second only to semantic errors in Friedman's symptom succession.

Several participants (RS, NS, AB, DB) showed an effect of spelling-sound consistency (when reading the Consistency $\times$ Imageability items from Monaghan \& Ellis, 2002), which is the cardinal symptom of surface dyslexia (Patterson \& Hodges, 1992). This is a somewhat surprising finding and one that few researchers have explored when investigating phonological/deep dyslexia. When further testing was undertaken using another assessment (Strain, Patterson, \& Seidenberg, 1995) the consistency effect disappeared in all but one participant $(\mathrm{AB})$. Error analysis of AB's responses revealed that out of a total of 21 errors on inconsistent items, only 3 were regularizations. Indeed, error analyses across all the participants' incorrect responses on inconsistent items revealed very few of the "classic" regularization errors normally associated with surface dyslexia.

\section{Underlying Phonological and Semantic Impairments}

Although previous studies have suggested a link between phonological impairment and phonological dyslexia, these investigations have not specified which 
Table 2. Reading Assessments

\begin{tabular}{|c|c|c|c|c|c|c|c|c|c|c|c|c|c|c|}
\hline & \multirow{2}{*}{$\begin{array}{l}\text { Maximum } \\
\text { Score }\end{array}$} & \multicolumn{12}{|c|}{ Participant } & \multirow{2}{*}{$\begin{array}{c}\text { Controls }^{a} \\
\text { (Normal Cutoff) }\end{array}$} \\
\hline & & $L R$ & $M M$ & $R J$ & $R S$ & $A B$ & NS & $M R$ & $B N$ & $T J$ & $P G$ & $T H$ & $D B$ & \\
\hline Age (years) & & 58 & 58 & 40 & 64 & 83 & 51 & 72 & 52 & 60 & 66 & 48 & 61 & \\
\hline Months postonset & & 156 & 120 & 18 & 31 & 14 & 96 & 28 & 42 & 68 & 28 & 31 & 6 & \\
\hline \multicolumn{15}{|l|}{ Word Reading } \\
\hline \multicolumn{15}{|l|}{ Imageability $\times$ Frequency list } \\
\hline All items & 96 & 13 & 25 & 36 & 42 & 64 & 66 & 69 & 70 & 71 & 73 & 86 & 88 & $\diamond$ \\
\hline High imageability & 32 & 11 & 21 & 24 & 26 & 29 & 31 & 31 & 30 & 28 & 28 & 32 & 31 & $\diamond$ \\
\hline Low imageability & 32 & 0 & 1 & 3 & 4 & 15 & 14 & 14 & 17 & 20 & 16 & 28 & 26 & $\diamond$ \\
\hline \multicolumn{15}{|l|}{ PALPA 31} \\
\hline High imageability & 40 & 16 & 21 & 24 & 25 & 33 & 37 & 37 & 34 & 39 & 31 & 40 & 39 & $39.9(39.4)$ \\
\hline Low imageability & 40 & 0 & 2 & 1 & 13 & 18 & 27 & 26 & 27 & 32 & 27 & 37 & 39 & $39.5(38.1)$ \\
\hline Words from the semantic battery & 30 & 10 & 20 & 8 & 24 & 22 & 29 & 24 & 30 & 28 & 27 & 29 & 29 & $\diamond$ \\
\hline \multicolumn{15}{|l|}{ Monaghan \& Ellis (2002) } \\
\hline Consistent & 36 & 3 & 4 & 9 & 25 & 29 & 28 & 27 & 24 & 25 & 27 & 32 & 36 & 35.8 \\
\hline Inconsistent & 36 & 1 & 4 & 10 & 13 & 21 & 19 & 23 & 17 & 20 & 21 & 31 & 29 & 34.8 \\
\hline \multicolumn{15}{|l|}{ PALPA 32: part of speech } \\
\hline Total & 80 & 3 & 7 & 17 & 30 & 57 & 47 & 66 & 70 & 64 & 58 & 74 & 79 & $79.7(78.5)$ \\
\hline Nouns & 20 & 1 & 4 & 5 & 7 & 12 & 14 & 16 & 19 & 18 & 17 & 18 & 20 & \\
\hline Verbs & 20 & 1 & 1 & 6 & 13 & 17 & 12 & 17 & 18 & 17 & 13 & 19 & 19 & \\
\hline Adjectives & 20 & 1 & 2 & 3 & 5 & 14 & 14 & 17 & 18 & 14 & 16 & 20 & 20 & \\
\hline Functors & 20 & 0 & 0 & 3 & 5 & 14 & 7 & 16 & 15 & 15 & 12 & 17 & 20 & \\
\hline $\begin{array}{l}\text { PALPA 34: morphological } \\
\text { complexity }\end{array}$ & 30 & 1 & 1 & 7 & 13 & 18 & 12 & 24 & 23 & 24 & 23 & 24 & 29 & $\diamond$ \\
\hline \multicolumn{15}{|l|}{ Nonword Reading } \\
\hline PALPA 36 & 24 & 2 & 0 & 2 & 8 & 13 & 7 & 8 & 11 & 2 & 9 & 6 & 14 & $22.9(19.9)$ \\
\hline Derived from CCT items & 30 & 0 & 0 & 1 & 7 & 10 & 4 & 4 & 7 & 3 & 7 & 6 & 21 & $29.3(27.4)$ \\
\hline $\begin{array}{l}\text { Derived from Imageability } \times \\
\text { Frequency list items }\end{array}$ & 48 & 0 & 0 & 0 & 9 & 16 & 3 & 4 & 8 & 0 & 4 & 11 & 19 & $45.7(42.9)$ \\
\hline
\end{tabular}

Scores shown in italic type are within the normal range.

${ }^{\mathrm{a}}$ Control data: where the figures are known they are given as follows: 1 st figure $=$ mean, bracketed figure $=$ cutoff at 2 standard deviation below the mean.

$\diamond$ indicates normal performance assumed to be at ceiling on these easy tasks.

PALPA = Psycholinguistic Assessments of Language Processing in Aphasia (Kay et al., 1992); CCT = Camel and Cactus Test (Bozeat et al., 2000).

aspects of the phonological deficit are critical. The participants' performance was assessed, therefore, across a range of nonreading phonological tasks with the expectation that some but not necessarily all of these would (a) highlight the patients' phonological impairment and (b) give us some insights with regard to what aspect of their phonological impairment is critical for reading (see below). The results are shown in Table 5. It is clear that their reading deficit was accompanied by impaired phonology in all cases. This deficit was most evident on the phonological manipulation tasks (Patterson \& Marcel, 1992) on which there was considerable variation across the case series. Further evidence of a general phonological deficit was provided by both rhyme judgment and production tasks. Word repetition was mildly impaired (in comparison 
Table 3. Reading Error Analysis

\begin{tabular}{|c|c|c|c|c|c|c|c|c|c|c|c|c|}
\hline & \multicolumn{12}{|c|}{ Participant } \\
\hline & $L R$ & $M M$ & $R J$ & $R S$ & $A B$ & NS & $M R$ & $B N$ & TJ & $P G$ & $T H$ & $D B$ \\
\hline Accuracy (total words $=388$ ) & 12.1 & 21.6 & 28.9 & 47.7 & 67.5 & 68.3 & 76.3 & 76 & 78.1 & 74 & 91 & 95.1 \\
\hline \multicolumn{13}{|l|}{ Type of error } \\
\hline Semantic & 5.9 & 4.9 & 4.6 & 0 & 0 & 1 & 0 & 0 & 0 & 0.3 & 0 & 0 \\
\hline Visually related words & 12.6 & 10.3 & 13.4 & 11.3 & 5.9 & 11.3 & 12.9 & 9 & 8.2 & 4.6 & 5.2 & 1.5 \\
\hline Visually related nonwords & 2.3 & 0.8 & 12.4 & 28.1 & 14.9 & 1.8 & 3.6 & 7.7 & 7.7 & 5.7 & 0.8 & 2.1 \\
\hline Morphological & 2.1 & 5.9 & 5.2 & 2.6 & 2.1 & 4.4 & 2.1 & 1.8 & 2.6 & 9 & 2.3 & 0.8 \\
\hline No response & 36.3 & 37.4 & 22.4 & 1.8 & 0 & 6.2 & 3.4 & 0.3 & 0.8 & 0.3 & 0 & 0 \\
\hline Other & 28.6 & 19.1 & 13.1 & 8.5 & 9.5 & 7 & 1.8 & 5.2 & 2.6 & 6.2 & 0.8 & 0.5 \\
\hline
\end{tabular}

This error analysis is based on all the words read by the participants (collapsing across tests, $n=388$ ). Reading accuracy and the rate of different error types are expressed as a percentage of trials.

with control subjects) for all but one of the cohort (LR), but scores on this task were generally close to ceiling. This probably reflects a relative insensitivity of word repetition to phonological impairments. Indeed, as the phonological tasks became more taxing, the participants' accuracy declined (phonological manipulation = rhyme production $<$ nonword repetition $<$ word repetition: $F(3,33)=30.4, p<.001)$.

In addition to phonological tests, the case series were assessed for the integrity of their semantic representations/comprehension (see Table 6). It is evident from these results that although performance was better on these semantic tests than on the phonological assessments, many of the participants demonstrated some degree of comprehension deficit. This was most evident on the various synonym judgment tests and the pictureassociation task. The participants' performance on the word-picture matching tasks was much closer to ceiling, which may reflect the fact that these tasks are relatively easy and insensitive to mild semantic impairments.

It is worth noting that 8 of the 12 participants were outside the normal range on the auditory minimal pair discrimination task (most notably RS and NS, see Table 7) suggesting some additional receptive/perceptu-

Table 4. Comparison of Patient Profiles to Friedman's Severity-based Symptom Succession

\begin{tabular}{lcccccccc}
\hline & $\begin{array}{c}\text { Word } \\
\text { Reading, } \\
n=96\end{array}$ & $\begin{array}{c}\text { Nonword } \\
\text { Reading, } \\
n=102\end{array}$ & $\begin{array}{c}\text { Derivational } \\
\text { Errors }\end{array}$ & $\begin{array}{c}\text { Visual } \\
\text { Errors }\end{array}$ & $\begin{array}{c}\text { Noun }> \\
\text { Functor }\end{array}$ & $\begin{array}{c}\text { Noun }> \\
\text { Verb }\end{array}$ & $\begin{array}{c}\text { Imageability } \\
\text { Effect }\end{array}$ & $\begin{array}{c}\text { Semantic } \\
\text { Errors }\end{array}$ \\
\hline $\mathbf{L R}^{\mathrm{a}}$ & $\mathbf{1 3}$ & $\mathbf{2}$ & $\checkmark$ & $\checkmark$ & $\checkmark$ & $\checkmark$ & $\checkmark$ & $\checkmark$ \\
$\mathbf{M M}^{\mathrm{a}}$ & $\mathbf{2 5}$ & $\mathbf{0}$ & $\checkmark$ & $\checkmark$ & $\checkmark$ & $\checkmark$ & $\checkmark$ & $\checkmark$ \\
$\mathbf{R J}^{\mathrm{a}}$ & $\mathbf{3 6}$ & $\mathbf{3}$ & $\checkmark$ & $\checkmark$ & $\checkmark$ & $\checkmark$ & $\checkmark$ & $\checkmark$ \\
$\mathrm{RS}^{\mathrm{b}}$ & 42 & 24 & $\checkmark$ & $\checkmark$ & $\checkmark$ & $\boldsymbol{x}$ & $\checkmark$ & $\boldsymbol{x}$ \\
$\mathrm{AB}^{\mathrm{b}}$ & 64 & 39 & $\checkmark$ & $\checkmark$ & $\boldsymbol{x}$ & $\boldsymbol{x}$ & $\checkmark$ & $\boldsymbol{x}$ \\
$\mathbf{N S}^{\mathrm{a}}$ & $\mathbf{6 6}$ & $\mathbf{1 4}$ & $\checkmark$ & $\checkmark$ & $\checkmark$ & $\checkmark$ & $\checkmark$ & $\checkmark$ \\
$\mathrm{MR}^{\mathrm{b}}$ & 69 & 16 & $\checkmark$ & $\checkmark$ & $\boldsymbol{x}$ & $\boldsymbol{x}$ & $\checkmark$ & $\boldsymbol{x}$ \\
$\mathrm{BN}^{\mathrm{b}}$ & 70 & 26 & $\checkmark$ & $\checkmark$ & $\boldsymbol{x}$ & $\boldsymbol{x}$ & $\checkmark$ & $\boldsymbol{x}$ \\
$\mathrm{TJ}^{\mathrm{b}}$ & 71 & 5 & $\checkmark$ & $\checkmark$ & $\boldsymbol{x}$ & $\checkmark$ & $\checkmark$ & $\boldsymbol{x}$ \\
$\mathbf{P G}^{\mathrm{a}}$ & $\mathbf{7 3}$ & $\mathbf{2 0}$ & $\checkmark$ & $\checkmark$ & $\checkmark$ & $\checkmark$ & $\checkmark$ & $\boldsymbol{x}$ \\
$\mathrm{TH}^{\mathrm{b}}$ & 86 & 23 & $\checkmark$ & $\checkmark$ & $\boldsymbol{x}$ & $\boldsymbol{x}$ & $\checkmark$ & $\boldsymbol{x}$ \\
$\mathbf{D B}^{\mathrm{b}}$ & $\mathbf{8 8}$ & $\mathbf{5 4}$ & $\checkmark$ & $\checkmark$ & $\boldsymbol{x}$ & $\boldsymbol{x}$ & $\boldsymbol{x}$ & $\boldsymbol{x}$ \\
\hline
\end{tabular}

${ }^{a}$ Expected pattern. Shown in boldface.

${ }^{\mathrm{b}}$ Unexpected pattern. 
Table 5. Phonology Assessments

\begin{tabular}{|c|c|c|c|c|c|c|c|c|c|c|c|c|c|c|}
\hline & \multirow{2}{*}{$\begin{array}{l}\text { Maximum } \\
\text { Score }\end{array}$} & \multicolumn{12}{|c|}{ Participant } & \multirow{2}{*}{$\begin{array}{c}\text { Controls }^{a} \\
\text { (Normal Cutoff) }\end{array}$} \\
\hline & & $L R$ & $M M$ & $R J$ & $R S$ & $A B$ & NS & $M R$ & $B N$ & $T J$ & $P G$ & $T H$ & $D B$ & \\
\hline Manipulation tasks & 96 & 67 & - & 11 & - & 71 & - & 16 & 50 & 19 & - & 47 & 61 & 91.1 \\
\hline Rhyme judgment & 48 & 40 & 31 & 40 & 24 & 46 & 48 & 34 & 42 & 41 & 38 & 41 & 43 & $47.8(47)$ \\
\hline Rhyme production & 24 & 15 & - & 13 & - & 10 & 15 & - & 11 & 9 & - & 9 & 13 & $22(17.3)$ \\
\hline \multicolumn{15}{|l|}{ Repetition } \\
\hline Word & 126 & 126 & 119 & 98 & 17 & 122 & 125 & 120 & 122 & 120 & 120 & 124 & 122 & 126 \\
\hline Nonword & 48 & 44 & 23 & 22 & 1 & 24 & 44 & 31 & 41 & 36 & 23 & 42 & 40 & $44.8(37.1)$ \\
\hline Delayed word & 96 & 78 & 52 & 40 & - & 54 & 68 & 79 & 64 & 46 & 73 & 93 & 85 & $94.7(89.8)$ \\
\hline Delayed nonword & 48 & 17 & 4 & 4 & - & 9 & 21 & 11 & 6 & 2 & 12 & 29 & 16 & $41.2(30)$ \\
\hline
\end{tabular}

${ }^{\mathrm{a}}$ Control data: where the figures are known they are given as follows: first figure $=$ mean, bracketed figure $=$ cutoff at 2 SD below the mean. Dashes (-) indicate assessment was discontinued after 10 items when the participant was clearly unable to continue. Scores shown in italic type are within the normal range.

al processing problems. These difficulties, however, do not provide a complete explanation for the impaired semantic performance given that comprehension deficits were not confined solely to the auditory modality.

\section{Predicting Reading Symptoms from Underlying Language Impairments}

Because the current literature on phonological-deep dyslexia is dominated by single case studies, evidence for the link between the participants' reading disorder and their more general language impairments has been limited to noting associations across cases (Patterson et al., 1996) or simulating core reading symptoms in nonreading tasks (see the next section, Eliciting Core Reading Symptoms in Nonreading Tasks; Patterson \& Marcel, 1992). Our case series, for the first time, allows a more formal exploration of the link between phonologicaldeep dyslexia and primary system impairments (a method that has been used extensively to demonstrate

Table 6. Semantic Assessments

\begin{tabular}{|c|c|c|c|c|c|c|c|c|c|c|c|c|c|c|}
\hline & \multirow{2}{*}{$\begin{array}{l}\text { Maximum } \\
\text { Score }\end{array}$} & \multicolumn{12}{|c|}{ Participant } & \multirow{2}{*}{$\begin{array}{l}\text { Controls }^{a} \\
\text { (Normal Cutoff) }\end{array}$} \\
\hline & & $L R$ & $M M$ & $R J$ & $R S$ & $A B$ & $N S$ & $M R$ & $B N$ & $T J$ & $P G$ & $T H$ & $D B$ & \\
\hline \multicolumn{15}{|l|}{ Graded synonym judgment } \\
\hline Auditory/concrete & 25 & 15 & 14 & 14 & 8 & 18 & 18 & 14 & 10 & 17 & 23 & 22 & 14 & $21(15)$ \\
\hline Written/concrete & 25 & 9 & 19 & 13 & 18 & 23 & 19 & 11 & 16 & 18 & 22 & 24 & 15 & $23.9(21.4)$ \\
\hline Auditory/abstract & 25 & 15 & 13 & 14 & 10 & 19 & 18 & 14 & 13 & 19 & 19 & 21 & 16 & $21(14)$ \\
\hline Written/abstract & 25 & 16 & 11 & 18 & 11 & 20 & 15 & 15 & 13 & 20 & 24 & 19 & 15 & $23.3(20)$ \\
\hline $\begin{array}{l}\text { Imageability } \times \text { Frequency } \\
\text { synonym judgment }\end{array}$ & 96 & 46 & 51 & 68 & 54 & 74 & 80 & 60 & 68 & 72 & 85 & 86 & 77 & $94.5(90.98)$ \\
\hline \multicolumn{15}{|l|}{ Shortened semantic battery: } \\
\hline SWPM & 30 & 29 & 29 & 29 & 21 & 30 & 29 & 29 & 25 & 28 & 30 & 30 & 30 & $\diamond$ \\
\hline WWPM & 30 & 27 & 28 & 29 & 29 & 29 & 29 & 30 & 24 & 29 & 30 & 29 & 30 & $\diamond$ \\
\hline Picture association & 30 & 20 & 22 & 23 & 26 & 24 & 22 & 22 & 15 & 17 & 27 & 28 & 25 & $27.6(24.8)$ \\
\hline Picture naming & 30 & 12 & 15 & 15 & 15 & 12 & 21 & 22 & 18 & 25 & 25 & 29 & 29 & $\diamond$ \\
\hline
\end{tabular}

SWPM $=$ spoken word-picture matching; WWPM $=$ written word-picture matching.

${ }^{a}$ Control data: where the figures are known they are given as follows: first figure $=$ mean, bracketed figure $=$ cutoff at 2 SD below the mean.

Scores shown in italic type are within the normal range.

$\diamond$ indicates normal performance assumed to be at ceiling on these easy tasks. 
Table 7. Orthographic and Phonological Perception

\begin{tabular}{|c|c|c|c|c|c|c|c|c|c|c|c|c|c|c|}
\hline & \multirow{2}{*}{$\begin{array}{l}\text { Maximum } \\
\text { Score }\end{array}$} & \multicolumn{12}{|c|}{ Participant } & \multirow{2}{*}{$\begin{array}{c}\text { Controls }^{a} \\
\text { (Normal Cutoff) }\end{array}$} \\
\hline & & $L R$ & $M M$ & $R J$ & $R S$ & $A B$ & NS & $M R$ & $B N$ & $T J$ & $P G$ & $T H$ & $D B$ & \\
\hline \multicolumn{15}{|l|}{ PALPA } \\
\hline Cross-case letter match & 52 & 52 & 52 & 52 & 51 & 52 & 52 & 52 & 52 & 52 & 51 & 52 & 52 & $51.96(51.5)$ \\
\hline Letter string match & 60 & 59 & 56 & 60 & 50 & 59 & 58 & 60 & 60 & 52 & 60 & 60 & 58 & $\diamond$ \\
\hline VOSP degraded letters & 20 & 19 & 19 & 20 & 19 & 14 & 18 & 20 & 20 & 18 & 20 & 18 & 18 & $18.8(16)$ \\
\hline ADA minimal pair discrimination & 40 & 40 & 31 & 36 & 22 & 30 & 18 & 31 & 36 & 39 & 37 & 39 & 40 & $39.4(37.5)$ \\
\hline
\end{tabular}

${ }^{a}$ Control data: where the figures are known they are given as follows: first figure $=$ mean, bracketed figure $=$ cutoff at 2 SD below the mean.

Scores shown in italic type are within the normal range.

$\diamond$ indicates normal performance assumed to be at ceiling on these easy tasks.

the link between semantic impairment, surface dyslexia and, an array of other language and nonlanguage deficits-see the Introduction). Our analyses targeted four core deficits associated with phonological-deep dyslexia: (1) poor nonword reading, (2) a lexicality effect (difference between real and nonword reading), (3) semantic paralexias (proportion of total errors), and (4) an imageability effect (difference between high- and lowimageability items). The degree of each dyslexic symptom was correlated against the various phonological and semantic measures (RS's data were excluded from the correlations with phonological scores given his profound auditory perception/word deafness; all $p$ values, onetailed). The results were as follows.

(1) Nonword reading: as predicted by Patterson and colleagues (Patterson et al., 1996; Patterson \& Marcel, 1992), nonword reading was correlated with general phonological skill. This relationship was strongest for the most sensitive phonological tasks-phonological blending and segmentation $(r=.56, p=.04)$-whereas the relationship with the other phonological measures was much weaker. Nonword reading was not related to any of the semantic scores.

(2) In contrast, the lexicality effect was not correlated with phonological scores but was with performance on some of the semantic tests. The size of the lexicality effect increased as the participants' synonym judgment scores improved (auditory graded synonyms: $r=.58$, $p=.03$; written graded synonyms: $r=.50, p=.05$; Imageability $\times$ Frequency synonyms: $r=.73, p=.004$ ). There were no correlations with word-picture matching or the picture-association task.

(3) Exactly the same pattern was found for the rate of semantic errors-no correlations with the phonological tasks but negative correlations with synonym judgment (Imageability $\times$ Frequency: $r=-.48, p=.06$ ).

(4) Interestingly, imageability effects were correlated with both phonological and semantic domains. The effect became larger as phonological skills diminished (e.g., phonological manipulation: $r=-.55, p=.04$ ) yet reduced with better scores on synonym judgment (e.g.,
Imageability $\times$ Frequency: $r=-.52, p=.04)$. Again, the correlations with the picture-based semantic tasks were nonsignificant.

It is important to consider this variation in correlations with the semantic tasks in more detail given that it may indicate in what way semantic representations are supporting word reading. Several factors might contribute to this variation. The pattern of correlations within the semantic tasks are revealing. Those involving a picture input correlate with each other (picture association and word-picture matching tasks: $r$ between .707 and $.873, p$ between .02 and $<.001)$ as do the synonym judgment tests $(r$ between .74 and .82 , $p$ between .006 and .001). The picture and word-only-based tasks do not, however, correlate with each other. There are three possible explanations for these differences. First, different concepts are probed in these tasks. The picturebased tests only tap into concrete concepts, whereas synonym judgments cover both concrete and abstract words. This possible difference does not seem to explain the variation in correlations, however, because when the abstract items are dropped from the synonym judgment test, then the correlations with the picture-based tasks are not improved and remain nonsignificant. The second possibility is that the synonym judgment tests are picking up on impairments of the interaction between verbal and central semantic representations (with any damage to these concepts being detected by the picture-based tests). The third and closely related possibility is that there is a central semantic deficit that is detected more readily by the verbal tasks but this impairment tends to be stabilized with a picture input because of the systematic relationships between pictures and meaning (Lambon Ralph \& Howard, 2000; Caramazza, Hillis, Rapp, \& Romani, 1990).

\section{Eliciting Core Reading Symptoms in Nonreading Tasks}

As noted in the Introduction, several previous studies have demonstrated phonological-deep dyslexic symp- 
toms in nonreading, phonological tasks consistent with the primary systems hypothesis (Beland \& Mimouni, 2001; Patterson \& Marcel, 1992). We explored this possibility by investigating the influence of delay on repetition. Even in immediate repetition the participants exhibited a significant lexicality effect, $t(11)=5.2$, $p<.001$, although this was augmented by delay: Lexicality $\times$ Delay: $F(1,10)=12.6, p=.005$. Overall performance and the size of the lexicality effect in delayed repetition were very similar to reading aloud itself (see Figure 1A). Imageability effects are strongly associated with deep dyslexia and are the penultimate symptom in Friedman's proposed succession. In immediate repetition, the participants demonstrated a small yet significant effect of imageability, $F(2,22)=4.85, p=.02$. This difference was magnified considerably by the insertion of a filled delay, Imageability $\times$ Delay: $F(2,22)=$ $8.8, p=.002$, and the size of the resulting effect again approached that observed in reading itself (see Figure $1 \mathrm{~B}$ ). This pattern is not observed when controls are asked to repeat after a delay (see Table 5). In summary, not only do these participants replicate the finding that phonological-deep dyslexia is accompanied by a generalized phonological impairment, but also the nature of their impairment in these nonreading tasks mirrors that found in reading (in fact, one of the deep dyslexic cases, $\mathrm{MM}$, did produce semantic errors in repetition once a delay was added: e.g., "wicket" $\rightarrow$ "cricket," "paint" $\rightarrow$ "emulsion," "necklace" $\rightarrow$ "brooch.”)

\section{DISCUSSION}

Patterson and Lambon Ralph (1999) suggested that acquired disorders of reading could be understood in terms of impairments to three primary systems (semantics, phonology, and vision). Previous case-series studies
Figure 1. (A) Comparison of immediate and delayed repetition with reading. (B) Imageability effects in repetition and reading.

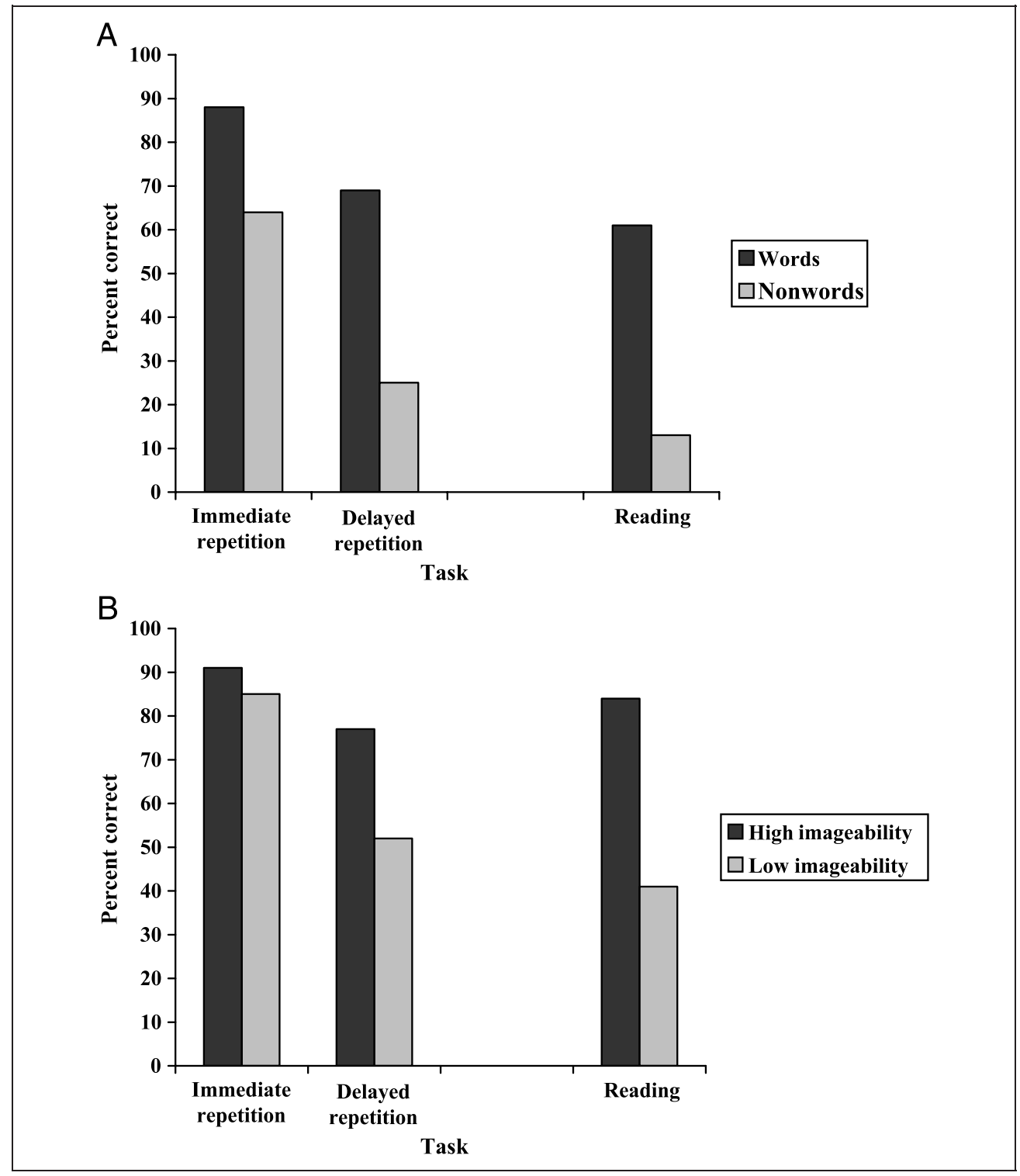


have demonstrated that semantic impairment leads to surface dyslexia and other predictable impairments in language and nonlanguage activities (Rogers et al., 2004; Lambon Ralph et al., 2001; Patterson et al., 2001; Graham et al., 2000; Patterson \& Hodges, 1992). For the first time, this study applied a similar case-series approach to explore the questions of whether phonological and deep dyslexia actually reflect a single type of acquired dyslexia (Friedman, 1996; Glosser \& Friedman, 1990) and second, whether this reflected more general phonological (Patterson \& Marcel, 1992) or semantic impairment (Friedman, 1996).

The results from this study strongly support the proposal that there is a link between phonological and deep dyslexia (Friedman, 1996; Glosser \& Friedman, 1990). From this perspective, deep dyslexia can be viewed as an extreme version of phonological dyslexia rather than a separate, independent disorder (Patterson \& Lambon Ralph, 1999). Despite considerable variation in word and nonword reading accuracy, there was substantial overlap in the dyslexic symptoms exhibited by these participants (including poor nonword reading, lexicality and imageability effects, visual and morphological paralexias). There was no sensible dividing line to separate the participants into distinct groups. Three of the participants with a classical deep dyslexic presentation (LR, RJ, and NS) did have a very severe nonword reading deficit, but their nonword reading was not completely abolished. This stands contrary to the notion that any sort of nonword reading ability will enable the individual to filter out semantic errors, thereby turning participants from deep into phonological dyslexics (Newcombe \& Marshall, 1980). Conversely, several of the participants at the milder end of the spectrum (notably PG and TH) had near-normal word reading, but they nonetheless produced derivational errors and exhibited an imageability effect in reading aloud. That so many of the participants demonstrated an imageability effect is striking in that this characteristic is strongly associated with deep rather than phonological dyslexia.

In addition to suggesting that phonological and deep dyslexia are linked, Friedman's (1996) review of previous cases indicated that there might be a particular severitybased succession of dyslexic symptoms (as laid out in Table 1). This review was hindered by the fact that the data were drawn from different single case studies with little or no overlap in assessment materials. Moreover, the participants represented readers of different languages (French and English). By using identical assessments across the same 12 participants, we were able to compare them directly. We found minimal evidence for a symptom succession in these cases. With the exception of semantic errors, the remaining symptoms were exhibited-to at least some degree-by most of the participants. Only semantic paralexias were confined to the poorest readers. In summary, it would appear that there is very little that qualitatively distinguishes phonological from deep dyslexia, but rather, these terms have come to denote quantitatively different points along a single dyslexia "continuum."

We were also able to use the case-series data to confirm that their reading disorder was directly related to the status of their phonological and semantic systemsand thereby reveal the factors that underpin the deepphonological continuum. Following Patterson and colleagues (Patterson et al., 1996; Patterson \& Marcel, 1992), we found that nonword reading accuracy was correlated with the participants' nonreading phonological skills. In addition, we found that three other core symptoms were related to the status of the participants' comprehension skills (only if measured by synonym judgment tasks). This is consistent with proposals by both Patterson et al. (1996) and Friedman (1996). Specifically, the participants' lexicality effect diminished with increasing semantic impairment. Similarly, the imageability effect and the rate of semantic errors increased in line with greater semantic deficits. Imageability effects also reduced as the participants' phonological scores improved. These findings would seem to reflect the intrinsically strong interaction between phonology and semantics (this interaction is, e.g., central to spoken comprehension and production: Lambon Ralph et al., 2002; Patterson \& Lambon Ralph, 1999). This suggests that words, more than nonwords, are more likely to be read correctly because the activation of phonology is boosted by their meaning (which nonwords, by definition, do not have). If these semantic representations or the interaction between semantics and phonology is affected, then the semantic boost for word reading is diminished and reading accuracy reduces to that observed for meaningless, pronounceable strings (nonwords). The negative correlations between semantic status and the imageability effect/semantic errors suggest that these symptoms reflect either pathological interaction with word meaning or interaction with pathological semantic representations. The mechanism would be identical to that proposed to account for the lexicality effect-the natural interaction between semantics and phonology. If semantic representations or their impact on phonology is intact then word reading will be boosted accurately. However, if the influence of semantics becomes disrupted then accurate reinforcement will be compromised-the extreme symptom of which is the production of semantic paralexias. Imageability effects actually reflect the status of the participants' phonological and semantic representations. Comprehension was graded by imageability for most of the participants and so any pathological influence of semantic representations on the activation of phonology was most likely for abstract words. At the same time, good phonological skills presumably protect reading aloud from the pathological influence of semantic representations. 
The fact that the "continuum" reflects a subtle interplay between phonological and semantic impairments is entirely consistent with the primary systems hypothesis (Patterson \& Lambon Ralph, 1999) and the triangle Parallel Distributed Processing (PDP) model of reading (Plaut et al., 1996). Both argue that normal and impaired reading reflect the interaction between a limited number of general systems. Performance of participants reflects a combination of the impairments themselves (e.g., impaired phonology) as well as the interaction between systems (e.g., phonological-semantic interactions). Although there may be other ways to explain the participants' reading data (see below), these approaches are distinguished by the fact that the reading disorder is assumed to arise from damage to systems that are not specific to one language activity but are utilized in a variety of cognitive domains. The ability to predict a range of dyslexia symptoms from the degree of impairment to phonology and semantics supports this approach. The primary systems hypothesis goes on to predict that, in addition, symptoms found in the reading domain should be found in nonreading tasks if probed for in the correct way (with the exception of symptoms uniquely associated with orthography, e.g., visual-orthographic reading errors). We added to previous evidence (Patterson \& Marcel, 1992) by investigating this possibility by using delayed repetition (see also Beland \& Mimouni, 2001). Even though immediate repetition is relatively insensitive to phonological impairment, the participants-as a group-demonstrated small yet significant effects of lexicality and imageability. When a filled delay was added, however, both of these effects became much more marked, with the size of each approaching that found in reading itself. The parallel between the participants' deep-phonological dyslexia and an emergent deep-phonological dysphasia is striking and strongly supports the primary systems explanation of acquired dyslexias.

The results of this study would suggest that the severity-based reading continuum between phonological and deep dyslexia is actually underpinned by two factors: phonological and semantic impairment. Is it sensible, therefore, to keep referring to this as a continuum when this term implies variation along a single dimension? We believe that this description has utility in two regards. First, as noted by Friedman and her colleagues, there is no sensible dividing line between phonological and deep dyslexia and the term continuum captures this true state of affairs. In addition, the term also embodies the notion that these patients reflect different severities of reading/language impairment. One can conceive of the phonological-to-deep range of patients occupying a swathe of positions within a two-dimensional space defined by phonology and meaning (see below). We note, here, that it might also be the case that substantial phonology-only impairments may compromise performance on semantic tests, including those that were most related to reading performance (verbal synonymjudgment tests). Such tasks require activation, maintenance, and manipulation of words in order to make decisions about the meaning of them. Severe phonological deficits may well impair this aspect of verbal comprehension tests. The triangle model of reading (Plaut et al., 1996) is based on a highly interactive system and, thus, severely impaired phonological representations alone may lead to semantic errors and imageability effects in reading. This could follow from the resultant, faulty phonological-semantic interactions or a compensatory overinvolvement of semantic representations in language activities. If correct, then the continuum might collapse onto a single, phonological dimension and thus explain the co-occurrence of impairments on semantic and phonological tests in the severe patients. Given the complexity of the interactions between semantic and phonological representations, the merit of this possibility will need to be tested in future implemented, computational models.

We have, so far, been silent on reading models other than the triangle model. This is because this study was motivated in part by the triangle model/primary systems hypothesis. It is entirely possible that the reading data could be modeled within a dual-route framework (Coltheart, Rastle, Perry, Langdon, \& Ziegler, 2001). Indeed, our explanation in terms of phonological and semantic impairments could be reformed into a proposal based on reading-specific mechanisms, such as the summation hypothesis (Friedman, 1996; Hillis \& Caramazza, 1995; Marshall \& Newcombe, 1973). The summation hypothesis proposes that reading performance reflects the conjoint action of lexical and nonlexical reading routes. Poor nonword reading follows from damage to the nonlexical reading route. Word reading is partially accomplished along the nonlexical reading route but is boosted by its interaction with the lexical pathway. This gives rise to the lexicality effect and, when damaged, might lead to imageability effects and semantic paralexias (especially when the remaining functioning of the nonlexical route is low or even nonexistent). Whereas it is entirely possible that a dual-route model could account for these and other phonological-deep dyslexia results, the approach is limited in two regards. First, it is difficult to think of ways in which to test for the integrity of each reading pathway that do not involve reading words and nonwords (i.e., avoiding the danger of circularity). In contrast, the triangle model predicts that reading should be related to the status of general cognitive systems. Given that these systems are nonspecific to reading, it is then possible to assess their status through other modalities. Secondly, given that the other approaches are based on reading-specific modules, these theories make few, if any, predictions with regard to the participants' performance in other domains. Unlike the primary systems hypothesis, they provide no explanation for why symptoms found in one 
domain (e.g., reading) might also be closely paralleled in others (e.g., repetition, phonological manipulation, etc.).

If we combine the results of this study with the analogous findings from the literature on the link between surface dyslexia and semantic impairment, then the complete picture suggested by the primary systems hypothesis is exposed. Figure 2 is a graphical summary of the relationship between the various acquired dyslexias and the underlying phonological and semantic impairments. A complete picture would actually require a third dimension to represent a visual-orthographic deficit and its link with pure alexia or letter-by-letter reading (Behrmann, Nelson, \& Sekuler, 1998; Behrmann, Plaut, \& Nelson, 1998). Normal reading is based on intact phonological and semantic representations, although variation in normal performance might reflect small, inherent individual differences along these same dimensions (Plaut, 1997). When phonology remains intact but semantic representations become impoverished-as is the case in semantic dementia-the pattern of surface dyslexia results (Patterson \& Hodges, 1992). In contrast, phonological-deep dyslexia occupies the opposite corner of this semantic-phonological space. With phonological impairment but little in the way of semantic deficits, a pattern close to mild phonological dyslexia would result. The data from this study suggest that poor nonword reading is directly related to the underlying phonological impairment, whereas lexicality effects are high because of the interaction with intact semantic representations (or intact interaction with semantic representations). Imageability effects and semantic errors become more pronounced once phonological impairment is accompanied by semantic impairment or disordered semantic-phonological interactions (thus, the deep end of the phonological-deep dyslexia contin-

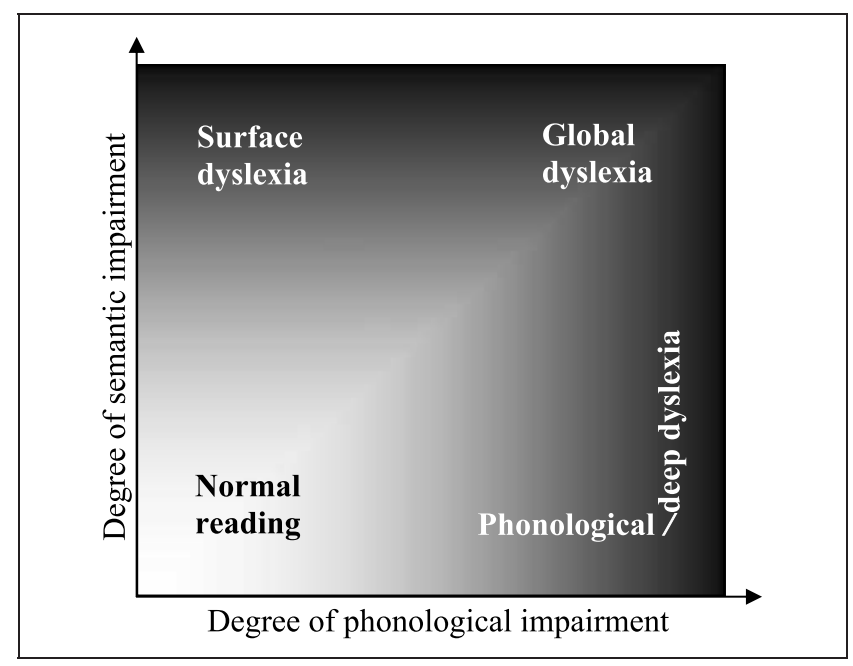

Figure 2. The positioning of the acquired dyslexias within a phonological-semantic space. uum is shown as curving around the corner of Figure 2 to combine phonological and semantic impairment). In the limit, severe semantic and phonological impairments would produce global dyslexia.

To finish, we note that when viewed in these terms, the principal nature and explanations for these reading disorders closely resembles the terms proposed for these acquired dyslexias by Shallice and Warrington: The link between semantic impairment and surface dyslexia was captured by the alternative term, semantic dyslexia (Shallice, Warrington, \& McCarthy, 1983), whereas the continuum of phonological-deep dyslexia and its association with phonological impairment was encapsulated by the term phonemic dyslexia (Shallice \& Warrington, 1975). Although we hope that the detailed case-series studies of our contemporary groups of semantic and phonemic dyslexics have extended and elucidated their relationship with more general underlying neural systems, the sense of familiarity with proposals from 30 years ago should not go unnoticed.

\section{METHODS}

\section{Participants}

Twelve participants with the symptoms of phonological or deep dyslexia were recruited via local speech and language therapy services. Possible participants were screened and recruited on the basis of demonstrating in reading aloud: (a) a lexicality effect, (b) an imageability effect, or (c) production of semantic paralexias. The first 12 participants who fulfilled these inclusion criteria were recruited and we thus avoided only including people with very marked deep dyslexic symptoms or relatively mild phonological dyslexia, which would reinforce the notion of disparate disorders. It is evident that the participants did represent a relatively wide range of severity of reading impairment. All the participants had acquired their dyslexia post-cerebrovascular accident, although this neurological diagnosis was not used as a method of selection. All were medically stable. Participants were 10 men and 2 women ranging in age from 40 to 83 years (mean age 59.4 years; $S D$ 11.4). Months postonset varied between 6 and 156 (mean 53 months; $S D$ 47.2). Unfortunately, information from structural scanning is limited, but left-hemisphere infarction was confirmed by computed tomography in eight cases.

\section{Assessments}

Each participant was asked to complete 29 assessments. These were conducted over 6-10 one-hour testing sessions. Control data were drawn from the original source of each test or when unavailable, 10 age-matched controls were asked to complete the tasks (noted below 
against the relevant tests). The following tests were included within the battery.

Section 1: Assessments to Explore the Possible Continuum Between Phonological and Deep Dyslexia

A range of reading tasks were administered to identify deep and surface dyslexic symptoms and to measure the severity of the participant's acquired dyslexia:

(1) Reading aloud matched lists of high- and lowimageability/frequency words: (a) a set of 96 items drawn from three levels of imageability and two levels of frequency, (b) a set of 80 items with two levels of imageability and two levels of frequency (Test 31: Kay et al., 1992).

(2) Reading aloud matched lists of words with varying syllable length. Participants read aloud the 30 words in the shortened semantic battery (see below) in which syllable length is varied but frequency and age of acquisition are controlled (one, two, and three or more syllables: Bozeat, Lambon Ralph, Patterson, Garrard, \& Hodges, 2000).

(3) Reading aloud matched lists of regular and irregular words. Two word lists were used to explore this variable: (a) a set of 72 items that varied consistency and imageability. These items were all low frequency and were matched for age of acquisition and other properties (Monaghan \& Ellis, 2002). (b) When a statistically significant consistency effect emerged using the above test, participants were asked to read a further matched set of 64 low-frequency items varied on consistency and imageability (Strain et al., 1995).

(4) Reading aloud words of different grammatical classes: (a) sets of 20 nouns, adjectives, verbs, and functors were presented. Items were matched for frequency and length; the nouns, verbs and adjectives were matched for imageability (Test 32: Kay et al., 1992). (b) A further retrospective investigation for any part-of-speech effect was carried out by examining reading accuracy in words of different grammatical classes across all the real word-reading tests used. (In cases where there was ambiguity about whether a written word should be treated as a noun or a verb, e.g., rake, the higher Celex lemma frequency was used to determine grammatical class.) A discrepancy between the findings of (a) and (b) is probably accounted for by the fact that the PALPA test is relatively stringently controlled and contains fewer items. There has been considerable debate in the literature over the reality of any part-of-speech effect, with some researchers arguing that part-ofspeech effects may, in fact, be an artifact of an imageability effect (because functors are generally lower in imageability than other word classes). We should emphasize that we have focused here on simply identifying the presence or absence of this effect, treating our data in the same way as Friedman does in her 1996 article. We are thus treating the part-of-speech effect as a symptom rather than as an incontrovertible effect.

(5) Reading aloud inflected/affixed words to elicit derivational errors. Participants read aloud 30 words (regularly inflected/irregularly inflected/with derivational endings) that were matched for the frequency and imageability of the stem (modified version of Test 34 : Kay et al., 1992).

(6) Nonword reading: (a) 24 monosyllabic nonwords varying in length from three to six letters (Test 36: Kay et al., 1992), (b) 30 nonwords derived from and matched (for letter length and numbers of orthographic and phonological neighbors) with the items in (2), (c) 48 nonwords derived from and matched (for letter length and numbers of orthographic and phonological neighbors) with the low-frequency items in 1(a). A group of 10 , age-matched controls were asked to read these various nonwords to provide normative data.

All error responses were analyzed and coded. Rates of these different errors (expressed as a percentage of the total number of trials) are shown in Table 3. Responses were categorized as visual/phonological errors when $50 \%$ of the letters in the response were present in the stimulus (following the criterion proposed by Morton \& Patterson, 1980). Ellis and Marshall (1978) showed that entirely random stimulus-response pairings produced an average "semantic" error rate of 9\%. Thus, for the purposes of accessing the features of Friedman's symptom succession, in order for a participant to be considered to be producing genuine semantic paralexias, we set a threshold of producing more than $10 \%$ semantic errors (as a percentage of all visually unrelated errors). Friedman's fixed-symptom succession was compared with the presence or absence of each dyslexia symptom in each individual participant. This binary analysis could be criticized for insensitivity to any variation in severity of each dyslexic symptom. Therefore, we conducted further analyses of the case-series data by fitting sigmoid functions to the severity of each of the symptoms across each of the participants. The results were unchanged.

\section{Section 2: Assessments to Explore the Possible Bases of a Continuum-Assessing Semantic and Phonological Impairments}

(1) Two synonym judgment tests were used: (a) A graded synonyms (Warrington, McKenna, \& Orpwood, 1998) test consisted of two 25-item subtests (concrete/ abstract), each psychometrically graded for difficulty. Participants were asked to choose which word is most similar in meaning to a given target (e.g., javelin: shield or spear). Items were spoken by the examiner or given as printed text on separate testing occasions. (b) In a second assessment, participants were asked to choose which of three written words was most similar in meaning to a written target (e.g., keep: become, save, put). Items were the same as those in 1(a), which were 
read aloud on a separate occasion-allowing a direct comparison between reading aloud and comprehension.

(2) Shortened semantic battery: word-picture matching (Bozeat et al., 2000). Thirty target pictures were presented with nine semantic foils. Participants were asked to pick the named picture. The test was administered in spoken or written forms on separate testing occasions.

(3) Shortened semantic battery: semantic association (Camel and Cactus Test: Bozeat et al., 2000). This task uses the same 30 concepts. It is an all-picture test in which participants were asked to identify which picture was most closely related in meaning to a given target (e.g., camel: tree, sunflower, cactus, rose). The four choices are drawn from the same semantic category.

(4) Shortened semantic battery: picture naming (Bozeat et al., 2000). Participants were asked to name the 30 concepts from black-and-white line drawings.

Given that reading and repetition are not the most sensitive measures of phonological impairment, these standard assessments were augmented with a variety of other tasks:

(1) Phonological manipulation tasks (Patterson \& Marcel, 1992): (a) segmentation-participants were asked to delete the first sound of 48 monosyllabic spoken stimuli and to say what remained. Half of the stimulus items and target responses were words and half were nonwords, yielding four conditions: word $\rightarrow$ word (e.g., vale $\rightarrow$ ale), nonword $\rightarrow$ word (e.g., vage $\rightarrow$ age), word $\rightarrow$ nonword (e.g., vane $\rightarrow$ ane) and nonword $\rightarrow$ nonword (e.g., vafe $\rightarrow$ afe); (b) assembly-on different testing occasions, participants were asked to add a phoneme onset to the "rhyme" of a monsyllabic spoken stimulus and say the result (e.g., /v/ + age $\rightarrow$ vage). The same items were used as in the segmentation test above, thus yielding the same four conditions.

(2) Rhyme judgment: Participants were asked to judge whether or not two spoken words rhyme.

(3) Rhyme production: Participants were asked to produce a word that rhymes with a given spoken word.

(4) Word and nonword repetition: Participants were asked to repeat a range of the words and nonwords that, on separate testing occasions, they had read aloud: (a) the same 96 words from Section 1: 1(a) and Section 2: 1(b) above (varying imageability and frequency); (b) the 30 words used in range of semantic assessments in Section 2: 2-4 above (varying length); (c) the 30 nonwords used in Section 1: 6(b) above; and (d) the 48 nonwords used in Section 1: 6(c) above.

(5) Delayed word and nonword repetition: Participants were required to repeat the items from 4(a) and (d) above with the insertion of a filled 5-sec delay (filled with rehearsal of their own name) between their immediate repetition and a second repetition.

The group of 10 age-matched controls again provided normative results for these tasks.
Three additional assessments were administered to test for basic orthographic and phonological recognition: (a) upper- and lowercase letter matching tasks (Tests 19-21: Kay et al., 1992); (b) visual recognition of degraded letters (Warrington \& James, 1991); (c) auditory discrimination of nonword minimal pairs (Franklin, Turner, \& Ellis, 1992).

\section{Acknowledgments}

We thank all the participants who contributed their time and energy to this study. The study was conducted while JC was supported by a midcareer award from The Health Foundation (ref: 2795/767). We thank David Howard for his support and advice when running this study.

Reprint requests should be sent to Matthew A. Lambon Ralph, School of Psychological Sciences, University of Manchester, Oxford Road, Manchester M13 9PL, UK, or via e-mail: matt.lambon-ralph@manchester.ac.uk.

\section{REFERENCES}

Beauvois, M., \& Derouesne, J. (1979). Phonological alexia: Three dissociations. Journal of Neurology, Neurosurgery and Psychiatry, 42, 1115-1124.

Behrmann, M., Nelson, J., \& Sekuler, E. (1998). Visual complexity in letter-by-letter reading: "Pure" alexia is not so pure. Neuropsychologia, 36, 1115-1132.

Behrmann, M., Plaut, D. C., \& Nelson, J. (1998). A literature review and new data supporting an interactive account of letter-by-letter reading. Cognitive Neuropsychology, 15, $7-52$.

Beland, R., \& Mimouni, Z. (2001). Deep dyslexia in the two languages of an Arabic/French bilingual patient. Cognition, 82, 77-126.

Berndt, R., Haendiges, A., Mitchum, C., \& Wayland, S. (1996). An investigation of nonlexical reading impairments. Cognitive Neuropsychology, 13, 763-801.

Bozeat, S., Lambon Ralph, M. A., Patterson, K., Garrard, P., \& Hodges, J. R. (2000). Non-verbal semantic impairment in semantic dementia. Neuropsychologia, 38, 1207-1215.

Caramazza, A., Hillis, A. E., Rapp, B. C., \& Romani, C. (1990). Multiple semantics or multiple confusions? Cognitive Neuropsychology, 7, 161-168.

Cipolotti, L., \& Warrington, E. (1995). Semantic memory and reading abilities: A case report. Journal of the International Neuropsychological Society, 1, 104-110.

Coltheart, M. (1996). Phonological dyslexia: Past and future issues. Cognitive Neuropsychology, 13, 749-762.

Coltheart, M., Rastle, K., Perry, C., Langdon, R., \& Ziegler, J. (2001). A dual route cascaded model of visual word recognition and reading aloud. Psychological Review, 108, 204-256.

Ellis, A. W., \& Marshall, J. (1978). Semantic errors or statistical flukes? A note on Allport's "on knowing the meaning of words we are unable to report." Quarterly Journal of Experimental Psychology, 30, 569-575.

Franklin, S., Turner, J., \& Ellis, A. W. (1992). The ADA Comprehension Battery: Action for Dysphasic Adults. York, UK: University of York.

Friedman, R. B. (1996). Recovery from deep alexia to phonological alexia: Points on a continuum. Brain $\mathcal{E}$ Language, 52, 114-128.

Glosser, G., \& Friedman, R. B. (1990). The continuum of deep/phonological dyslexia. Cortex, 26, 343-359. 
Graham, K. S., Hodges, J. R., \& Patterson, K. E. (1994). The relationship between comprehension and oral reading in progressive fluent aphasia. Neuropsychologia, 32, 299-316.

Graham, N. L., Patterson, K., \& Hodges, J. R. (2000). The impact of semantic memory impairment on spelling: Evidence from semantic dementia. Neuropsychologia, 38, $143-163$.

Hillis, A. E., \& Caramazza, A. (1995). Converging evidence for the interaction of semantic and sublexical phonological information in accessing lexical representations for spoken output. Cognitive Neuropsychology, 12, 187-227.

Hodges, J. R., Patterson, K., Oxbury, S., \& Funnell, E. (1992). Semantic dementia: Progressive fluent aphasia with temporal lobe atrophy. Brain, 115, 1783-1806.

Jefferies, E., Lambon Ralph, M. A., Jones, R., Bateman, D., \& Patterson, K. (2004). Surface dyslexia in semantic dementia: A comparison of the influence of consistency and regularity. Neurocase, 10, 290-299.

Joanisse, M. F., \& Seidenberg, M. S. (1999). Impairments in verb morphology after brain injury: A connectionist model. Proceedings of the National Academy of Sciences, U.S.A., 96, 7592-7597.

Kay, J., Lesser, R., \& Coltheart, M. (1992). Psycholinguistic Assessments of Language Processing in Aphasia (PALPA). Hove, UK: Erlbaum.

Lambon Ralph, M. A. (2004). Reconnecting cognitive neuropsychology: Commentary on 'Harley's Does cognitive neuropsychology have a future?' Cognitive

Neuropsychology, 21, 31-35.

Lambon Ralph, M. A., \& Graham, N. L. (2000). Previous cases: Acquired phonological and deep dyslexia. Neurocase, 6, 141-178.

Lambon Ralph, M. A., \& Howard, D. (2000). Gogi aphasia or semantic dementia? Simulating and assessing poor verbal comprehension in a case of progressive fluent aphasia. Cognitive Neuropsychology, 17, 437-466.

Lambon Ralph, M. A., McClelland, J. L., Patterson, K., Galton, C. J., \& Hodges, J. R. (2001). No right to speak? The relationship between object naming and semantic impairment: Neuropsychological evidence and a computational model. Journal of Cognitive Neuroscience, 13, 341-356.

Lambon Ralph, M. A., Moriarty, L., \& Sage, K. (2002). Anomia is simply a reflection of semantic and phonological impairments: Evidence from a case series study. Aphasiology, 16, 56-82.

Marshall, J., \& Newcombe, F. (1973). Pattern of paralexia: A psycholinguistic approach. Journal of Psycholinguistic Research, 2, 175-200.

Monaghan, J., \& Ellis, A. W. (2002). What, exactly, interacts with spelling-sound consistency in word naming? Journal of Experimental Psychology: Learning, Memory, and Cognition, 28, 183-206.

Morton, J., \& Patterson, K. (1980). A new attempt at an interpretation, or, an attempt at a new interpretation. In M. Coltheart, K. Patterson, \& J. Marshall (Eds.), Deep dyslexia (pp. 91-118). London: Routledge \& Kegan Paul.

Newcombe, F., \& Marshall, J. (1980). Transcoding and lexical stabilisation in deep dyslexia. In M. Coltheart, K. Patterson, \&
J. Marshall (Eds.), Deep dyslexia (pp. 176-188). London: Routledge \& Kegan Paul.

Patterson, K. (1982). The relation between reading and phonological coding: Further neuropsychological observations. In A. W. Ellis (Ed.), Normality and pathology in cognitive functioning (pp. 77-111). London: Academic Press.

Patterson, K., \& Behrmann, M. (1997). Frequency and consistency effects in a pure surface dyslexic patient. Journal of Experimental Psychology: Human Perception and Performance, 23, 1217-1231.

Patterson, K., \& Hodges, J. R. (1992). Deterioration of word meaning: Implications for reading. Neuropsychologia, 30, $1025-1040$.

Patterson, K., \& Lambon Ralph, M. A. (1999). Selective disorders of reading? Current Opinion in Neurobiology, 9 , 235-239.

Patterson, K., Lambon Ralph, M. A., Hodges, J. R., \& McClelland, J. L. (2001). Deficits in irregular past-tense morphology associated with degraded semantic knowledge. Neuropsychologia, 39, 709-724.

Patterson, K., Lambon Ralph, M. A., Jefferies, E., Woollams, A., Jones, R., Hodges, J. R., et al. (2006). "Pre-semantic" cognition in semantic dementia: Six deficits in search of an explanation. Journal of Cognitive Neuroscience, 18, 169-183.

Patterson, K., \& Marcel, A. (1992). Phonological ALEXIA or PHONOLOGICAL alexia? In J. Alegria, D. Holender, J. Junca de Morais, \& M. Radeau (Eds.), Analytic approaches to buman cognition. Amsterdam: Elsevier.

Patterson, K., Susuki, T., \& Wydell, T. N. (1996). Interpreting a case of Japanese phonological alexia: The key is in phonology. Cognitive Neuropsychology, 13, 803-822.

Plaut, D., McClelland, J. L., Seidenberg, M. S., \& Patterson, K. (1996). Understanding normal and impaired word reading: Computational principles in quasi-regular domains.

Psychological Review, 103, 56-115.

Plaut, D. C. (1997). Structure and function in the lexical system: Insights from distributed models of word reading and lexical decision. Language and Cognitive Processes, 12, 765-805.

Rogers, T., Lambon Ralph, M. A., Hodges, J. R., \& Patterson, K. (2004). Natural selection: The impact of semantic impairment on lexical and object decision. Cognitive Neuropsychology, 21, 331-352.

Shallice, T., \& Warrington, E. (1975). Word recognition in a phonemic dyslexic patient. Quarterly Journal of Experimental Psychology, 27, 187-199.

Shallice, T., Warrington, E. K., \& McCarthy, R. (1983). Reading without semantics. Quarterly Journal of Experimental Psychology, 35, 111-138.

Strain, E., Patterson, K., \& Seidenberg, M. S. (1995). Semantic effects in single-word naming. Journal of Experimental Psychology: Learning, Memory, and Cognition, 21, 1140-1154.

Warrington, E., \& James, M. (1991). The Visual Object and Space Perception Battery. Bury St Edmunds: Thames Valley Test Company.

Warrington, E., McKenna, P., \& Orpwood, L. (1998). Single word comprehension: A concrete and abstract word synonym test. Neuropsychological Rehabilitation, 8, 143-154. 